\title{
VCSEL-Based Atmospheric Trace Gas Sensor Using First Harmonic Detection
}

\author{
Lijuan Lan ${ }^{\circledR}$, Jia Chen ${ }^{\circledR}$, Member, IEEE, Xinxu Zhao, and Homa Ghasemifard
}

\begin{abstract}
This paper presents a measurement system based on the first harmonic in tunable diode laser absorption spectroscopy using a vertical-cavity surface-emitting laser to measure the atmospheric $\mathrm{CO}_{2}$ and $\mathrm{H}_{2} \mathrm{O}$ concentrations. The developed system separates the residual amplitude modulation signal from the harmonics and then eliminates it. A digital signal processing is developed to autonomously infer the wavelength and light intensities of the laser. The gas concentrations are determined without extra calibration. The long-term measurements are taken to validate the precision and accuracy of the system. Based on the Allan variance analysis, the broad wavelength scanning enhances the measurement precision, and the first-harmonic detection can achieve about two times as high precision as the traditional second-harmonic detection. The field measurements implemented in early spring of 2018 in Munich were compared with the commercial nondispersive infrared (NDIR) sensor. The outcomes have revealed that our system has high accuracy for the gas concentration measurements and high consistency with the NDIR sensor. The diurnal variations of $\mathrm{CO}_{2}$ concentration have demonstrated that $\mathrm{CO}_{2}$ concentration in urban areas is affected by the biosphere and meteorological conditions and the daily anthropogenic activities. Furthermore, the air trajectory analysis based on the HYSPLIT model has found that the $\mathrm{CO}_{2}$ emission sources primarily come from the southeast of Munich. The developed system described in this paper has a great potential for in situ trace gas concentration measurements, the analysis on the polluted gas distributions, and the verification of the pollutant transport model in urban areas.
\end{abstract}

Index Terms-Trace gas concentration, tunable diode laser absorption spectroscopy (TDLAS), wavelength modulation spectroscopy (WMS), first harmonic, vertical-cavity surface-emitting laser (VCSEL).

G REENHOUSE gases (GHG) have a significant negative impact on the climate change. Research has indicated that carbon dioxide $\left(\mathrm{CO}_{2}\right)$, one of the dominant GHG, has

Manuscript received January 18, 2019; revised February 22, 2019; accepted February 23, 2019. This work was supported in part by the Institute for Advanced Study, Technical University of Munich, funded by the German Excellence Initiative and the European Union Seventh Framework Program under Grant 291763 and in part by the China Scholarships Council through the Program under Grant 201608080018. The associate editor coordinating the review of this paper and approving it for publication was Dr. Ioannis Raptis. (Corresponding authors: Lijuan Lan; Jia Chen.)

L. Lan, J. Chen, and X. Zhao are with the Department of Electrical and Computer Engineering, Technical University of Munich, 80333 Munich, Germany (e-mail: lijuan.lan@tum.de; jia.chen@tum.de).

H. Ghasemifard is with the Professorship of Ecoclimatology Department, Technical University of Munich, 85354 Freising, Germany.

Digital Object Identifier 10.1109/JSEN.2019.2901793 increased by about 2 ppm (parts per million) per year in recent years. The cities and their surroundings sustain around $54 \%$ of the global population and contribute nearly $70 \%$ of the total anthropogenic $\mathrm{CO}_{2}$ emissions [1]. Therefore, continuously measuring the $\mathrm{CO}_{2}$ concentration is of great significance for controlling the carbon emissions and studying the diffusion of the pollutant in urban areas [2]-[4].

Tunable diode laser absorption spectroscopy (TDLAS) is a typical method for the in-situ trace gas measurements since the laser scans across the absorption line at an extremely small bandwidth, around $30 \mathrm{MHz}$ or smaller, which is about one-tenth of the absorption linewidth [5]-[7]. Unlike the direct TDLAS is employed when absorbance is above $1 \%$, the wavelength modulation spectroscopy (WMS) has wide applications in the trace gas measurements (absorbance less than 1\%) and the harsh environments [8], [9]. The theory and mathematical definitions of WMS are outlined in Appendix A. However, due to the arbitrary variation in the phase shift in wavelength, the concentration-dependent harmonics are assigned to $\mathrm{X}$ and $\mathrm{Y}$ axes in the lock-in amplifier (LIA) at random. Hence, to obtain the complete harmonics, the most common methods are either manually setting the phase of reference signal or using the magnitude of two axes. The former method is inconvenient, while the latter is widely employed in applications [10]. The phase shift in wavelength is discussed in Appendix A.

The harmonics produced by the LIA consist of the absorption line shape and gas parameters information [11], [12]. The concentration-independent residual amplitude modulation (RAM) deforms the harmonics especially the first harmonic. In the well-known "calibration-free $n f / 1 f$ " method, the RAM in the first harmonic is used to normalize the other harmonics [13]-[15]. Nevertheless, the RAM is acquired from the absorption-free condition, it should be characterized carefully prior to the measurement or from a separate laser beam which makes the measurement inconvenient and complex [10]. Additionally, W. Johnstone et al. used the RAM to recover the absorption line shape based on the phasor decomposition method. However, this method is valid under small modulation index $(m<0.5)$ when only the first harmonic is utilized, which leads to low harmonic signal-to-noise ratio (SNR); nonetheless, it can be used for any $m$ values if the tuning coefficient is given and higher harmonics are employed [16]-[19]. An external optical fiber and the balanced detection were applied to null RAM in the intensity modulated experiment, which increased the complexity in operation and signal 
processing [20]-[22]. Hangauer et al. [11] utilized multiharmonic to reconstruct the transmission and Peng et al. [23] employed odd-harmonic to recover the absorbance shape, the $m$ was extended to higher values $(m>2.0)$ in their approaches.

Compared with the conventional distributedfeedback (DFB) laser, the vertical-cavity surface-emitting laser (VCSEL) has many unique features, such as low power consumption, fast and wide wavelength tuning range to cover several absorption lines. Thus, the wide tuning VCSEL can be used to simultaneously measure different gases [24]-[27]. The first harmonic has the highest SNR among all harmonics, thus, theoretically it should have a highest precision in gas sensing. However, due to the strong RAM, the first harmonic is distinctly deformed as a non-linear offset with a gradient particularly in the wide tuning situations (see black line in Fig. 4(b)). Therefore, the magnitude of $\mathrm{X}$ and $\mathrm{Y}$ components in the first harmonic cannot be employed directly to infer the gas concentration. It is crucial to build up a simple system and eliminate the RAM in the continuous gas measurement when using the first harmonic detection.

In J. Chen and A. Hangauer's research, the broad scanning range was employed for the wavelength calibration while the narrow scanning was for gas concentration measurements using the linear least square curve fitting (LLSCF) algorithm [28], [29]. They concluded that the second harmonic detection had higher precision than the first harmonic detection [11]. On the contrary, Lan et al. [30] focused on using the wide tuning VCSEL multi-harmonic for gas concentration measurement and found that the first harmonic could achieve better performance than the second harmonic in gas detection. It has shown that multi-harmonic detection including first harmonic can enhance the precision of the system. However, the previous study did not focus on the removal of RAM in first harmonic and the development of a compact system using first harmonic for urban GHG measurements.

This article focuses on utilizing the first harmonic for atmospheric gas sensing based on the VCSEL's wide tuning range. A measurement system was developed to autonomously eliminate the RAM signal in the harmonics, calibrate the wavelength and laser light intensities, and measure the in-situ $\mathrm{CO}_{2}$ and $\mathrm{H}_{2} \mathrm{O}$ concentrations. Then, Allan-Werle Variance Analysis was deployed to analyze the precision of our measurement system. The field measurement compared with the commercial nondispersive infrared (NDIR) sensor was carried out in early spring of 2018 in Munich. The analysis on the $\mathrm{CO}_{2}$ diurnal variation demonstrated that the $\mathrm{CO}_{2}$ concentration in urban areas is affected not only by biosphere and meteorological conditions but also by the daily anthropogenic activities. The backward trajectory analysis based on HYSPLIT model was implemented to identify $\mathrm{CO}_{2}$ sources in urban areas.

\section{EXPERIMENTAL FOUNDATION}

\section{A. First Harmonic Detection}

The basic principle of WMS has been introduced in several articles [11], [12], [31]. According to Eqs. 6 and 8 in Appendix A, for optically thin samples (absorbance $\ll 10 \%$ ), $A_{0} \approx 1$ and $A_{1}, A_{2}, A_{3}, A_{4}, \cdots \ll A_{0}$, which are assumed as 0 . Thus,

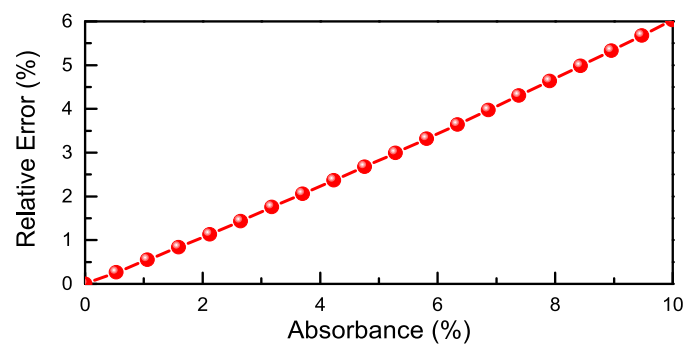

Fig. 1. The error analysis of concentration measurement when the $S_{0 f}$ is served as $S_{0 f} \approx \overline{I_{0}}$ to normalize the harmonics.

the zeroth harmonic picked out via LIA is: $S_{0 f}=I_{0 \theta}^{\mathrm{X}} \approx \overline{I_{0}}$. The $S_{0 f}$ can be served as the central light intensity $\left(\overline{I_{0}}\right)$ when the absorbance is weak [32].

When normalized with respect to $S_{0 f}$, the $\mathrm{X}$ and Y components of first harmonic, $X_{1 f}$ and $Y_{1 f}$, are simplified as:

$$
\left\{\begin{array}{l}
X_{1 f}=A_{1}+\frac{I_{1}}{\overline{I_{0}}} \cos \psi \\
Y_{1 f}=\frac{I_{1}}{\overline{I_{0}}} \sin \psi .
\end{array}\right.
$$

It can be learned from Eq. 1 that $X_{1 f}$ consists of two parts: the harmonic signal $\left(A_{1}\right)$, which includes gas parameters, and the cosine $\psi$ of RAM; while $Y_{1 f}$ is concentration-independent and only contains the sine $\psi$ of RAM. The $\psi$ can be determined when $Y_{1 f}, \overline{I_{0}}$ and $I_{1}$ are given. Then, the measured $\psi$ is employed to eliminate RAM when it is substituted into the $X_{1 f}$ expression:

$$
\overline{X_{1 f}}=X_{1 f}-\frac{Y_{1 f}}{\tan \psi}=A_{1}=P S(T) L c H_{1},
$$

where $H_{1}$ are the first order Fourier components of the spectral line shape. Thus, the $\overline{X_{1 f}}$ is pure concentration-dependent and can be used to infer gas concentration when $P$ and $T$ are given:

$$
c=\frac{\overline{X_{1 f}}}{P S(T) L H_{1}} \text {. }
$$

From Eq. 3, the gas concentration can be obtained without the extra calibration against the standard reference gas. Thus, this method gets rid of the calibration proces.

Figure 1 is the error analysis of the concentration measurement when $S_{0 f}$ is used as $\bar{I}_{0}$ to normalize the harmonic signals. As can be seen from Fig. 1, the relative errors increase as the increment of the absorbance. To ensure the measurement error within $1 \%$, the absorbance should not exceed $2 \%$. Therefore, for the trace gas measurement when absorbance $\ll 10 \%$, the approximation of $S_{0 f} \approx \overline{I_{0}}$ will not introduce a large measurement error.

\section{B. Experimental Setup}

The diagram of the experimental setup is shown in Fig. 2. A VCSEL (VL-2004-1-SQ-A5) [33] is used as the light source whose wavelength is controlled by a laser driver (Arroyo Instruments 6301). The absorption signal is reflected by a concave mirror with $75 \mathrm{~mm}$ of focus length and collected into an InGaAs amplified photodetector (PD, PDA10DT-EC). Subsequently, the detected signal is recorded and digitized 


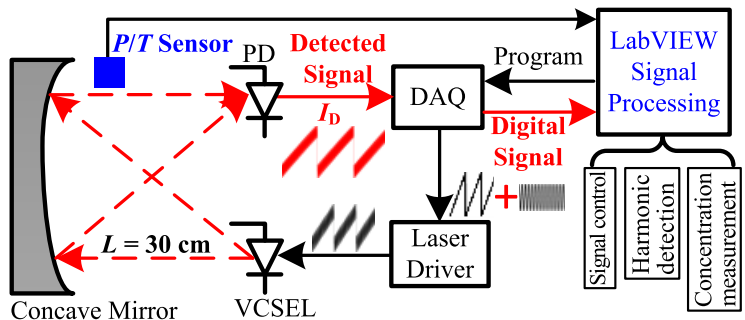

Fig. 2. Experimental setup for trace gas concentration measurement.

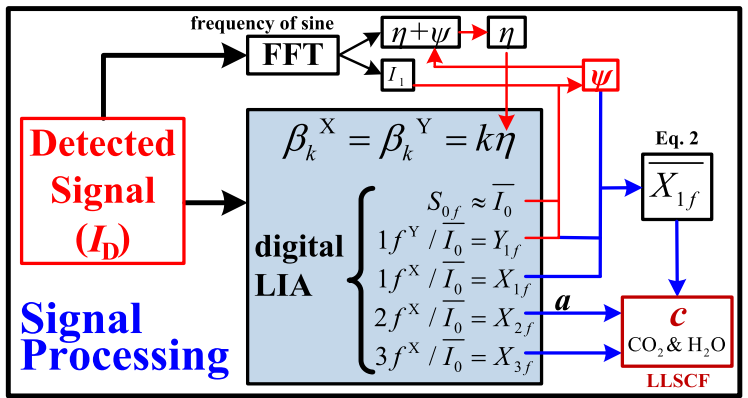

Fig. 3. Signal processing of harmonic detection and gas concentration measurement. The red arrows are the process to determine $\psi$ in an iterative procedure. The shade part is the digital lock-in amplifier (LIA).

via a high-speed memory data acquisition card (DAQ, NI USB-6361, resolution: 16 bits, and sampling rate: $2 \mathrm{MS} / \mathrm{s}$ ). The digitized data is sent into the signal processing to further calculate gas concentration. In this process, the DAQ is deployed not only to record the detected signal but also as a signal generator to provide the ramp and sinusoidal signals such that the laser wavelength is modulated. A sensor using the Raspberry Pi system is set nearby to synchronously measure $P$ and $T$. The signal processing is explained in the following Sect. II-C.

\section{Autonomous Measurement System}

A digital signal processing (LabVIEW) is developed to calculate the intermediate parameters as mentioned in Sect. II-A and Appendix A, and to obtain gas concentrations. The first part of the processing is the signal controlling and acquisition. The LabVIEW programs DAQ to generate the low-frequency $\operatorname{ramp}(10 \mathrm{~Hz}, 1.2 \mathrm{~V})$ and high-frequency sinusoidal $(6 \mathrm{kHz}$, $60 \mathrm{mV}$ ) signals to modulate the VCSEL in a wide range, and then to record the absorption signal into LabVIEW, which is shown as black line in Fig. 4(a). In this case, the modulation indices $(m)$ of each line are around 3.0 which ensures the high SNR of the first, second and third harmonics (Fig. 5, Appendex B).

The second part is the harmonic detection. As indicated in Fig. 3, a digital LIA is developed to produce the zeroth to third harmonics. Note that the zeroth harmonic can be produced in our developed LIA by setting the frequency of the reference signal to 0 . The red dotted line in Fig. 4(a) is the central light intensity $\left(\overline{I_{0}}\right)$. In WMS, the zeroth harmonic $\left(S_{0 f}\right)$ can be treated as: $S_{0 f} \approx \overline{I_{0}}$ when the absorbance is weak (absorbance $\ll 10 \%)$. The $S_{0 f}$ is averaged from the 10 sequential scans $(10 \mathrm{~Hz})$ to improve SNR and used to normalize the other harmonics. Figure 4(b) exhibits the curves of the normalized $X_{1 f}$ and $Y_{1 f}$. The peak positions of each absorption lines (the
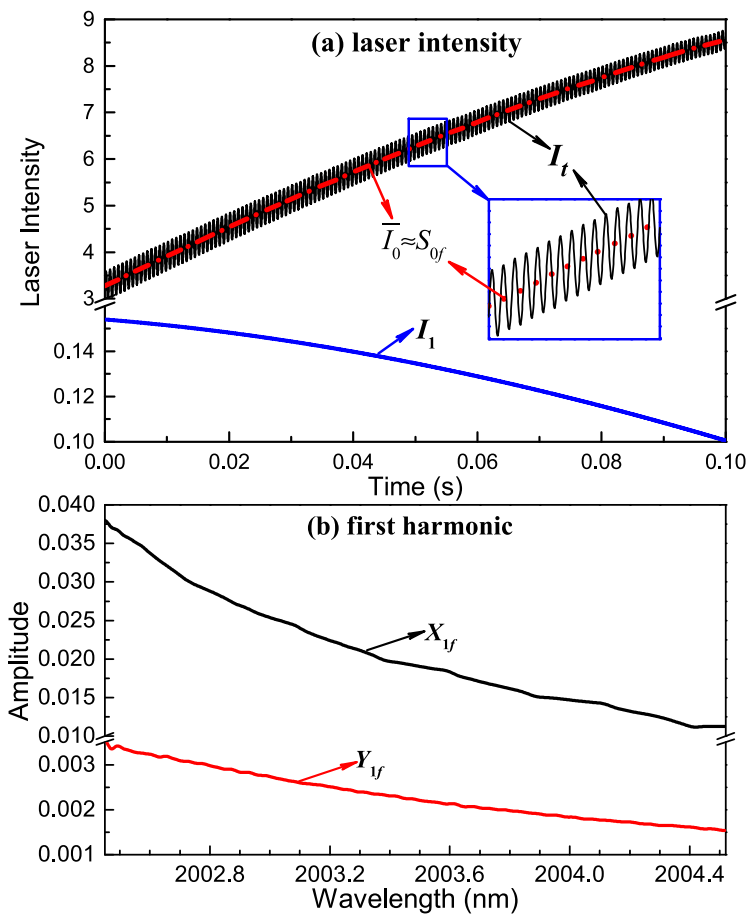

Fig. 4. (a) The detected signal via the photodetector $\left(I_{t}\right)$ and its central light intensity ( $\overline{I_{0}}$, red dotted line). The $S_{0 f}$ can be served as $S_{0 f} \approx \overline{I_{0}}$ when the absorbance is weak. The modulation intensity $\left(I_{1}\right)$ is obtained from an FFT subroutine. (b) The normalized $\mathrm{X}$ and $\mathrm{Y}$ components of first harmonic in a scanning cycle, where $X_{1 f}$ includes the RAM.

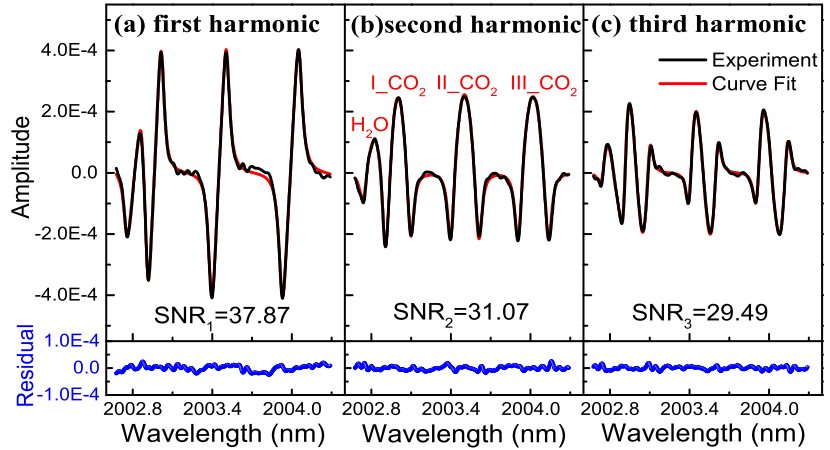

Fig. 5. Wide-scanned concentration-dependent harmonic signals obtained from Fig. 4(a); (a) first harmonic without RAM, (b) second harmonic, and (c) third harmonic. The red lines are the calculation curves based on the LLSCF. The bottoms are the residuals of curve fit results. The concentrations of $\mathrm{CO}_{2}$ and $\mathrm{H}_{2} \mathrm{O}$ are $411.8 \mathrm{ppm}$ and $5.56 \%$, respectively.

absorption line centers are $2002.829 \mathrm{~nm}\left(\mathrm{H}_{2} \mathrm{O}\right), 2002.998 \mathrm{~nm}$, $2003.503 \mathrm{~nm}$, and $2004.019 \mathrm{~nm}$, respectively) in the normalized $X_{2 f}$ (Fig. 5(b)) are picked as the absorption line centers, thus, the wavelength versus the ramp signal is calibrated and the modulation depths $(a)$ are determined (Appendix C). A Fast Fourier Transform (FFT) subroutine is established to measure the modulation intensity $\left(I_{1}\right.$, as blue line in Fig. 4(a)) and light intensity phase $(\eta+\psi)$ when the extracted frequency is set the same as sine signal $(6 \mathrm{kHz})$. An iterative procedure is created to determine the phase shift $(\psi)$ as follows: a very first $\psi_{1}=0^{\circ}$ is used to subtract $\psi$ in the phase $(\eta+\psi)$ and get $\eta_{1}$. Then, the phase of the reference signal is set as $\beta_{1}^{\mathrm{Y}}=\eta_{1}$ to receive $Y_{1 f}$. Afterwards, according to $\overline{I_{0}}, I_{1}$ and $Y_{1 f}$, a new $\psi_{2}$ is obtained. The process is reiterated until $\psi$ is constant. In our system, when the sine frequency is $6 \mathrm{kHz}, \psi=6.7^{\circ}$. 
Thereby, when given $\overline{I_{0}}, I_{1}, \psi$, and $\eta$, the $X_{k f}$ and $Y_{k f}$ are obtained by setting the reference phase signal to: $\beta_{k}^{\mathrm{X}}=\beta_{k}^{\mathrm{Y}}=$ $k \eta$ (see Appendix A). The concentration-dependent $\overline{X_{1 f}}$ is acquired according to Eq. 2.

In the third part, the harmonics with different scanning ranges are extracted to infer gas concentrations based on the LLSCF algorithm [34], [35]. The spectral parameters of the absorption lines are acquired from the HITRAN databases and utilized for calculating $H_{k}$ [36]. The curve fitting results of the first, second and third harmonics with three $\mathrm{CO}_{2}$ absorption lines and one $\mathrm{H}_{2} \mathrm{O}$ absorption line are displayed in Fig. 5. The residuals in the bottom are in an order of $1 \times 10^{-5}$, indicating the high accuracy of the system.

Throughout the process, the wavelength and light intensities, and the harmonics are autonomously calibrated in the absence of the standard optical components such as etalon, beam splitter, and the absorption-free laser path. The entire procedure takes about $0.5 \mathrm{~s}$ which enables the real-time measurement. As Eq. 3 demonstrates, the gas concentrations are obtained without the standard reference gas. Therefore, this system is an autonomous calibration system for in-situ trace gas measurements. The absorbance of about $2 \%$ for the GHG sensing ensures that the assumption of $S_{0 f} \approx \overline{I_{0}}$ is valid in the system. The $P$ and $T$ compensations are carried out in the measurements. The air-broadening is dominant while the self-broadening uncertainty $(<1 \%)$ can be ignored in Pressure Broadening in the trace gas measurements [15]. Furthermore, the $\overline{I_{0}}$ is on-the-fly measured in every scanning cycle, the fluctuations of the light intensity can be instantaneously eliminated during the measurement. This system thus can be utilized even in vibration situations where the laser path is difficult to fix.

Using VCSEL, the wide tuning range which covers several gas species in a cycle enables not only the wavelength calibration but also several gas concentrations to be measured simultaneously. From Fig. 5, the first harmonic without RAM has the highest magnitude and highest SNR of 37.87, meaning that it has the best noise performance among all harmonics. However, compared the black line in Fig. 4(b) and the curves in Fig. 5(a), which included and excluded the RAM, the RAM in the original first harmonic is a distinct non-linear offset with gradient that submerges the harmonic signal. The RAM is complicated and difficult to determine in the wide tuning VCSEL. Consequently, it is important for VCSEL to eliminate RAM in the wide tuning range for first harmonic detection. Furthermore, the second order modulation intensity $\left(I_{2}\right)$ is much smaller than $I_{1}\left(0.3 \%\right.$ of $\left.I_{1}\right)$ so that the RAM in the second harmonic can be treated as a slope and an offset, as presented in [29]. The higher order modulation intensities can be neglected since they are even smaller.

\section{EXPERIMENTAL RESUlt AND ANALysis}

\section{A. Allan-Werle Variance Analysis}

Allan-Werle Variance is a method to identify and quantify the different noise terms that exist in the inertial sensor data, and calculate the detection limit of the sensor in the time domain [37], [38]. As displayed in Fig. 6, when the Allan Deviation $\left(\sigma_{\text {Allan }}\right)$ is minimum $\left(\sigma_{\text {Allan }}^{\min }\right)$, the optimum integrating time $\left(\tau_{\mathrm{opt}}\right)$ and the detection limit are determined. Allan
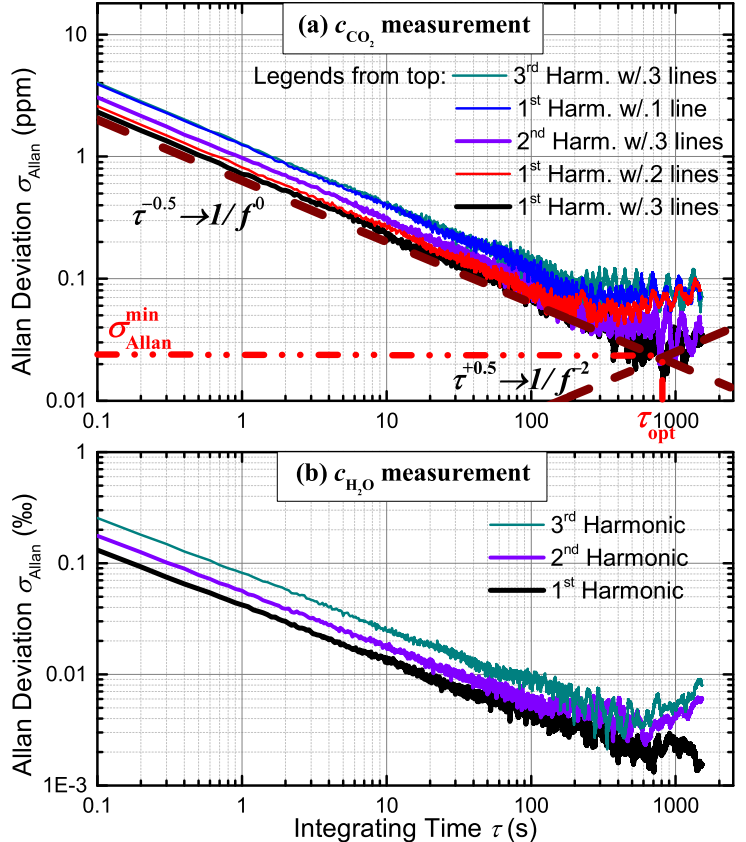

Fig. 6. Allan Deviations of the first, second and third harmonics with different absorption lines for $\mathrm{CO}_{2}$ and $\mathrm{H}_{2} \mathrm{O}$ concentration measurements. The winered lines represent slopes of -0.5 and 0.5 , which correspond to the power densities $S(f)=f^{0}$ (white noise) and $S(f)=f^{-2}$ (Brownian noise), respectively.

TABLE I

Allan Analysis For $\mathrm{CO}_{2} \& \mathrm{H}_{2} \mathrm{O}$ Concentration Measurements

\begin{tabular}{|c|c|c|c|c|}
\hline Gas & $S_{n f}$ w/. lines & $\sigma_{\text {Allan }} @ 1$ s & $\sigma_{\text {Allan }} @ 1$ min & $\sigma_{\text {Allan }}^{\min } @ \tau_{\text {opt }}(\mathbf{s})$ \\
\hline \multirow{5}{*}{$\mathrm{CO}_{2}$} & $1^{\text {st }}$ w/. 1 & $1.24 \mathrm{ppm}$ & $0.16 \mathrm{ppm}$ & $0.07 \mathrm{ppm} @ 300$ \\
\hline & $1^{\text {st }} \mathrm{w} / .2$ & $0.81 \mathrm{ppm}$ & $0.11 \mathrm{ppm}$ & 0.06ppm@300 \\
\hline & $1^{\text {st }} w / .3$ & $0.72 \mathrm{ppm}$ & $0.10 \mathrm{ppm}$ & 0.027 ppm@800 \\
\hline & $2^{\text {nd }} w / .3$ & $0.97 \mathrm{ppm}$ & $0.12 \mathrm{ppm}$ & 0.05ppm@400 \\
\hline & $3^{\text {rd }} \mathrm{w} / .3$ & $1.27 \mathrm{ppm}$ & $0.18 \mathrm{ppm}$ & 0.09ppm@200 \\
\hline \multirow{3}{*}{$\mathrm{H}_{2} \mathrm{O}$} & $1^{\text {st }} w / .1$ & $0.042 \%$ & $0.006 \%$ & $0.002 \% o @ 600$ \\
\hline & $2^{\text {nd }} w / .1$ & $0.057 \%$ & $0.007 \%$ & 0.004\%o@400 \\
\hline & $3^{\mathrm{rd}} \mathrm{w} / .1$ & $0.082 \%$ & $0.011 \%$ & 0.005\%o@200 \\
\hline
\end{tabular}

Deviations for $\mathrm{CO}_{2}$ and $\mathrm{H}_{2} \mathrm{O}$ concentration measurements are demonstrated in Fig. 6 and Table I. The measurement is performed in a stable climate chamber with the surrounding conditions of temperature: $296 \pm 0.5 \mathrm{~K}$ and pressure: $0.95 \pm$ $0.01 \mathrm{~atm}$, and $\mathrm{CO}_{2}$ and $\mathrm{H}_{2} \mathrm{O}$ concentrations of $466.8 \pm 0.2 \mathrm{ppm}$ and $5.05 \pm 0.01 \%$, respectively. From Fig. 6(a), the plots of the first harmonic detection illustrate that the more absorption lines are included, the higher precise the measurement is. That is, compared with the measurement result of the single absorption line (II_CO $\mathrm{CO}_{2}$, as annotated in Fig. 5(b)), the first harmonic detection with double (II and III_CO $\mathrm{CO}_{2}$ ) and triple absorption lines (I, II and III_CO $\mathrm{CO}_{2}$ ) have lower deviations. For example, the $\sigma_{\text {Allan }}$ of the double and the three absorption lines are $0.81 \mathrm{ppm}$ and $0.72 \mathrm{ppm}$ with $1 \mathrm{~s}$ averaged, respectively; while the $\sigma_{\text {Allan }}$ of the single line is $1.24 \mathrm{ppm}$. It concludes that the first harmonic detection with extended scanning range can improve the measurement precision.

When the RAM effect is eliminated, the first harmonic detection has higher precision than that of the second and third harmonic detections. That is, the Allan plots of the first harmonic have lower white and Brownian noise levels during the entire integrating time. Taking the $\mathrm{H}_{2} \mathrm{O}$ measurement as 
an example, the first harmonic achieves $6 \times 10^{-3} \%$ of the deviation with $1 \mathrm{~min}$ averaged, while the second and the third harmonic detections only obtain $7 \times 10^{-3} \%$ ond $0.011 \%$ o with the same integrating time, respectively. In terms of the detection limits, the first harmonic with three absorption lines obtains $0.027 \mathrm{ppm}$ when the $\tau_{\mathrm{opt}}$ is $800 \mathrm{~s}$ for $\mathrm{CO}_{2}$ concentration measurement, while the $\sigma_{\text {Allan }}^{\min }$ of the second harmonic is only $0.05 \mathrm{ppm}$ with $400 \mathrm{~s}$ averaged. In $\mathrm{H}_{2} \mathrm{O}$ detection, the $\sigma_{\text {Allan }}^{\min }$ of the first harmonic is $2 \times 10^{-3} \%$ with 600 s averaged, while the $\sigma_{\text {Allan }}^{\min }$ of the second harmonic is $4 \times 10^{-3} \%$ o with 400 s averaged. That is to say, the first harmonic detection has about two times better precision than the traditional second harmonic detection. As shown in Fig. 4(b), the primitive first harmonic curve is deformed in the wide scanning range because of the existence of the RAM. The harmonic signal is even submerged in the RAM and is hard to use for the concentration measurement. However, when the RAM signal is removed, the first harmonic detection has the best performance among all harmonics. Therefore, the elimination of RAM in the first harmonic is necessary and can effectively improve precision in gas sensing.

In short, the Allan Deviation Analysis demonstrates that when the RAM signal has been eliminated, the first harmonic detection has the highest precision in the gas concentration measurement. It can enhance the precision by nearly double when compared with the traditional second harmonic detection. That is because the first harmonic signal has the largest magnitude and SNR among all harmonics. As shown in Fig. 5, the SNR of the first harmonic is 37.87 which is higher than the second harmonic (31.07). The higher the order of the harmonic is, the lower the amplitudes and SNR are. Therefore, the low-order harmonics can achieve better precision performance in measurement. Widening the scanning range can not only measure various gas species simultaneously, but also can obtain higher precision because more information in the absorption lines are utilized. The experimental results also indicate the high stability of the measurement system.

\section{B. Accuracy of the Measurement}

The comparison experiments are implemented to verify the accuracy of our developed system, where a commercial NDIR sensor (Li-Cor $840 \mathrm{~A} \mathrm{CO}_{2} / \mathrm{H}_{2} \mathrm{O}$ gas analyzer) is served as a standard instrument as in [30]. However, due to the frequency instability of the optical filter which is controlled by the piezo, the NDIR sensor must be calibrated periodically according to the application and utilization frequency [39]. The inlet gas tube of the NDIR sensor is set close to the optical path of the TDLAS system. These two sensors are operated separately and simultaneously to acquire $\mathrm{CO}_{2}$ and $\mathrm{H}_{2} \mathrm{O}$ concentrations.

Figure 7 illustrates the comparison results of $\mathrm{CO}_{2}$ and $\mathrm{H}_{2} \mathrm{O}$ concentrations dating from March 30 to April 11, 2018. The outcomes of TDLAS are retrieved from the first harmonic detection with $10 \mathrm{~min}$ averaged for which the $\sigma_{\text {Allan }}$ are $0.03 \mathrm{ppm}$ for $\mathrm{CO}_{2}$ concentration and $0.02 \%$ for $\mathrm{H}_{2} \mathrm{O}$ concentration, as illustrated in Fig. 6 and Table I. From Fig. 7, we can learn that the measured concentrations from the TDLAS system is closely aligned with the values measured by the NDIR sensor, which reveals that these two sensors have a high consistency with each other. As shown in the

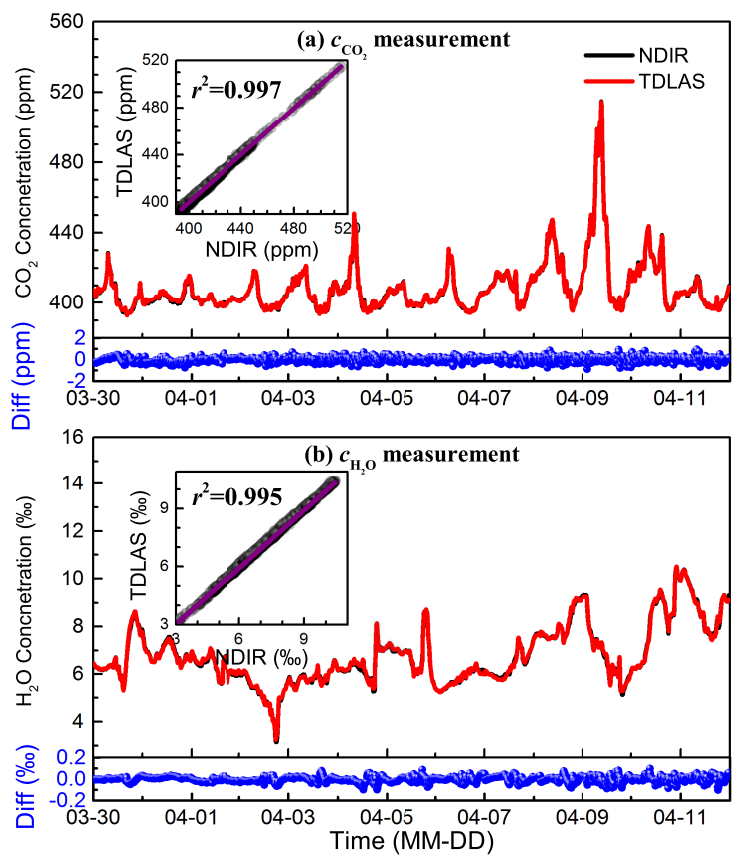

Fig. 7. $\mathrm{CO}_{2}$ and $\mathrm{H}_{2} \mathrm{O}$ concentrations measured by TDLAS and NDIR sensors, the experimental data is averaged over 10 minutes.

bottom of the plots, the differences between these two sensors are less than $1 \mathrm{ppm}$ for $\mathrm{CO}_{2}$ concentration and $0.1 \%$ for $\mathrm{H}_{2} \mathrm{O}$ concentration (much less than $1 \%$ relative), indicating the high accuracy of the developed system. Moreover, the linear regressions between the TDLAS and NDIR sensors are also demonstrated in Fig. 7, where the regression ranges are $390-520 \mathrm{ppm}$ with absorbance of $1.6 \times 10^{-3}-2.1 \times 10^{-3}$ for $\mathrm{CO}_{2}$ and $3-11 \%$ with absorbance of $5 \times 10^{-4}-1.8 \times 10^{-3}$ for $\mathrm{H}_{2} \mathrm{O}$, respectively. The correlation coefficients $\left(r^{2}\right)$ of above 0.99 demonstrate the high coherence of the two systems.

Overall, the wide scanning first harmonic signal can be utilized for measuring $\mathrm{CO}_{2}$ and $\mathrm{H}_{2} \mathrm{O}$ concentrations when the RAM is eliminated. The experimental results have confirmed that the first harmonic detection has high accuracy in gas sensing and has high consistency with the NDIR sensor. Furthermore, compared with the multi-harmonic detection, the sole first harmonic detection achieves a more stable measurement because less information is employed in the signal processing. The results have indicated that the first harmonic detection is more favorable in data processing and has wider applications in real-time GHG measurement [30].

\section{Diurnal Variation Analysis}

To provide a better understanding about the $\mathrm{CO}_{2}$ concentration variation in urban areas, the measurement results depicted in Fig. 7 are separated over days and presented in Fig. 8, where the foggy days from April 8 through 10 are excluded in the discussion. During the measurement period, the forsythia was blooming, meaning that most of the plant leaves had just started sprouting and that the vegetation photosynthesis and respiration from the biosphere were still not very strong. As displayed in Fig. 8(a), the diurnal cycle is clearly discernible. However, compared with the daily cycles in our previous work in [30], the lowest $\mathrm{CO}_{2}$ concentration in the study period 


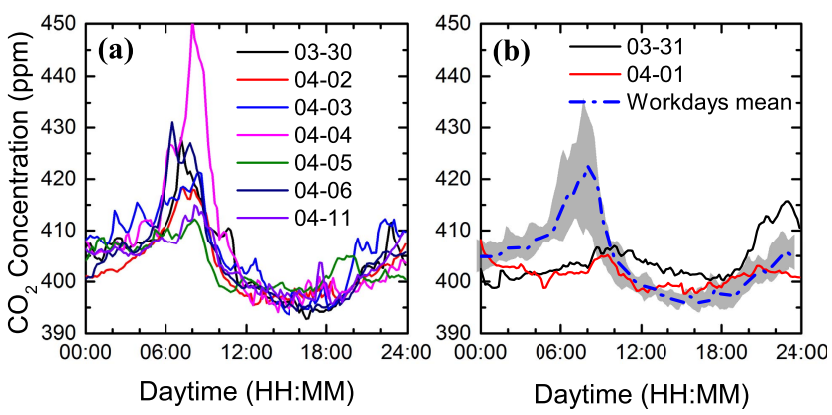

Fig. 8. Daily analysis of the $\mathrm{CO}_{2}$ concentration measurement in (a) workdays and (b) weekends; the dash-dotted line in (b) is the averaged value of (a), the gray part displays the $68 \%$ confidence interval.

(395 ppm) is higher than that in September (380 ppm) but lower than that in December (above $400 \mathrm{ppm}$ ). That is because the photosynthesis in early spring is weaker than that in September but stronger than that in December in Munich. Moreover, the averaged atmospheric temperature during the study period (about $8{ }^{\circ} \mathrm{C}$ ) is lower than in September $\left(15^{\circ} \mathrm{C}\right)$ but higher than in December $\left(0^{\circ} \mathrm{C}\right)$, leading to the height of the planetary boundary layer (PBL) stay between the heights of these two months. The sun heats up the ground surface in the day, thus the PBL increases while the $\mathrm{CO}_{2}$ concentration decreases. At night, the opposite occurs: the drop of the PBL increases the $\mathrm{CO}_{2}$ concentration [40].

The ground-based $\mathrm{CO}_{2}$ concentration is also influenced by the anthropogenic activities. Compared with the data measured on weekends, peak curves can be found in the morning from 06:00 to 10:00 on the workdays in Fig. 8(a). This is likely caused by rush-hour traffic increases between these hours, which results in increasing the $\mathrm{CO}_{2}$ concentration. On the weekends, when the weather was fair, there was no rush-hour peak at these hours, as can be seen in Fig. 8(b) [41]. This phenomenon indicates that the human activities have a great influence on the $\mathrm{CO}_{2}$ concentration in urban areas. Not only the special events like the festival celebrations in our previous study, but also the daily activities have a noticeable influence on the air quality in urban areas [30]. The above analysis demonstrates that the diurnal variation of $\mathrm{CO}_{2}$ concentration in urban areas is an integrative role combining the vegetation photosynthesis and respiration, the PBL (atmospheric temperature), and the daily anthropogenic activities of that area.

\section{Air Trajectory Analysis}

Nowadays, combining measurements with simulations is a conventional way to study and control carbon emissions in urban areas. The HYSPLIT (Hybrid Single Particle Lagrangian Integrated Trajectory) model is a common method to establish locations of emission sources from the receptor site [42]. Combined with the measured gas concentration at the time when the air parcel arrives at the measurement site, the air backward trajectories depict the air movement and gas distribution on the calculated paths. The PSCF (Potential Source Contribution Function) calculates the probability that describes the spatial distribution of probable source locations. The PSCF value is given as: $\mathrm{PSCF}=m_{i j} / n_{i j}$ in which $n_{i j}$ is the total number of trajectories that pass through the cell $(i, j)$ and $m_{i j}$ is the number of trajectories resulting in gas concentration
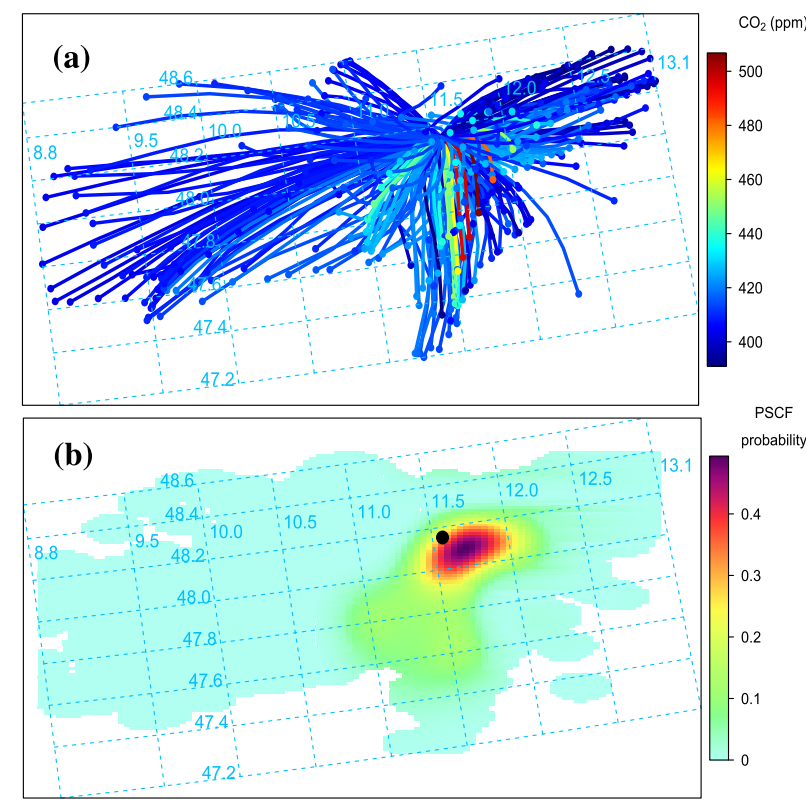

Fig. 9. (a) Six-hour backward air trajectories combined with the measured $\mathrm{CO}_{2}$ concentration (Fig. 7). (b) PSCF plot with over $75^{\text {th }}$ percentile $\left(\mathrm{CO}_{2}: 420 \mathrm{ppm}\right)$; the black circle is the measurement site.

that greater than a specific threshold $\left(75^{\text {th }}\right.$ percentile of $\mathrm{CO}_{2}$ concentration in the study, $420 \mathrm{ppm}$ ) [43], [44].

As illustrated in Fig. 9, the backward trajectories are calculated hourly combined with $\mathrm{CO}_{2}$ concentration ending at the measurement site (receptor) $\left(48.151^{\circ} \mathrm{N}\right.$ latitude and $11.568^{\circ} \mathrm{E}$ longitude) during the whole measurement period (Fig. 7) and then processed by the Openair package in R [45]. Six-hour backward trajectories are selected because the time is sufficient to determine the probable locations of both local and regional sources in urban areas [46]. The trajectories are divided into $0.05^{\circ} \times 0.05^{\circ}$ cells and the PSCF plot is smoothed as shown in Fig. 9(b), where the black circle is the position of the measurement site located at Munich. The cells are colored according to the PSCF values, with the dark-red part illustrating the highest probability of the source location. The measurement site is in the north of Munich city center, where the Munich Central Station, the Old Town and shopping malls locate at its southeast and are within a radius of $5 \mathrm{~km}$.

As indicated in Fig. 9(a), the air mass was primarily from the east, south and west directions, and few occurrence of air was from the north during the study period. The air from the south direction was much more polluted than that from east and west. From Fig. 9(b), we can learn that the darkest part is in the southeast of the measurement site, demonstrating the main emission source from this area in the period, which corresponds to the district with a high population density and high $\mathrm{CO}_{2}$ emissions. The preliminary air trajectory analysis has exhibited that when combined with the air trajectory model, the measurement results can be used for emission assessment. Therefore, our developed system can be applied in studying the $\mathrm{CO}_{2}$ distribution and predicting the pollutant emission sources in urban areas.

\section{CONCLUSION}

In conclusion, this paper developed a measurement system to autonomously eliminate the RAM in the first harmonic, 
and measure the gas concentrations using the broad scanning range VCSEL. At first, the method of first harmonic detection was proposed. Then, a digital signal processing pipeline was introduced to autonomously eliminate the RAM, calibrate the wavelength and light intensities, and infer gas concentrations. Subsequently, the $\mathrm{CO}_{2}$ and $\mathrm{H}_{2} \mathrm{O}$ measurements were implemented in both the stable environment and the field areas to verify the precision and accuracy of the developed system. According to the Allan-Werle Variance Analysis, widening the wavelength tuning range can enhance the precision of the developed system. The precision of the first harmonic detection was nearly doubled compared with the traditional second harmonic detection. The field measurements carried out in early spring of 2018 in Munich were compared with the commercial NDIR sensor. The results indicated that our developed system displayed high accuracy and had high consistency with the NDIR sensor. Further analysis revealed that the diurnal variations of the atmospheric $\mathrm{CO}_{2}$ concentration in urban areas are the comprehensive combination of the biosphere and meteorological conditions, and the local daily anthropogenic activities. The backward air trajectories were calculated based on the HYSPLIT model and PSCF method, the preliminarily results have shown that the $\mathrm{CO}_{2}$ emission sources mainly come from the southeast of Munich. According to the experimental and analysis results, the developed system presented in this article has a great potential for measuring the in-situ GHG concentrations, analyzing the polluted gas distributions, and validating the pollutant transport model in urban areas.

\section{APPENDIX A \\ THEORY OF WMS}

Wavelength modulation spectroscopy (WMS) is a method for sensitive absorbance measurements which uses a significantly faster sinusoidal signal riding on a slowly varying diode laser injection current. Instead of the transmission spectrum, the harmonic signals are obtained via the LIA. Due to its advantages of efficient noise suppression, for example, insusceptible to $1 / f$ noise, WMS enables to increase the sensitivity, precision and SNR in the trace gas measurements and the harsh environments. In TDLAS-WMS, when the laser is tuned by the low-frequency ramp and high-frequency sinusoidal signals, the instantaneous wavenumber and light intensity are expressed as:

$$
\left\{\begin{array}{l}
v(t)=\bar{v}+a \cos (\omega t+\eta) \\
I_{0}(t)=\overline{I_{0}}+I_{1} \cos (\omega t+\eta+\psi),
\end{array}\right.
$$

where $\bar{v}\left[\mathrm{~cm}^{-1}\right]$ and $\overline{I_{0}}$ denote the central wavenumber and central light intensity, they are the wavenumber and intensity of the central laser emission (without the sinusoidal modulation) which implemented by the slow ramp signal; $a\left[\mathrm{~cm}^{-1}\right]$ is the modulation depth and the modulation index is $m=a / \gamma$ where $\gamma$ is the linewidth; $I_{1}$ is the modulation intensity; $\omega$ $[\mathrm{rad} / \mathrm{s}]$ is the modulation angular frequency; $t[\mathrm{~s}]$ is the time; $\psi$ is the phase shift between the wavelength and intensity modulation; and $\eta$ is the phase shift in wavelength with respect to the tuning current. The $\eta$ is an arbitrary value in each measurement since the start recording point of the waveform is unfixed. The light intensity phase is $(\eta+\psi)$ and $\theta$ is defined as $\theta=\omega t+\eta$. Based on the Beer-Lambert Law, the transmitted light intensity $\left(I_{\mathrm{D}}\right.$, detected) is:

$$
\begin{aligned}
I_{\mathrm{D}}(t) & =I_{0}(t) \exp [-P S(T) L c \varphi(v(t))] \\
& =\sum_{k=0}^{\infty} I_{k \theta}^{\mathrm{X}} \cos k \theta-\sum_{k=0}^{\infty} I_{k \theta}^{\mathrm{Y}} \sin k \theta,
\end{aligned}
$$

where $P$ [atm], $S(T)$ [cm $-2 / \mathrm{atm}], T[\mathrm{~K}], L[\mathrm{~cm}]$, and $c$ are the total gas pressure, line strength, temperature, absorbing path length, and gas concentration, respectively; $\varphi[\mathrm{cm}]$ is the line shape which is a function of $v(t)$ and can be expressed as a Voigt profile [47]; $I_{k \theta}^{\mathrm{X}}$ and $I_{k \theta}^{\mathrm{Y}}$ are the $k$-th $(k=0,1,2,3, \cdots)$ Fourier components of the transmitted light intensity:

$$
\begin{cases}I_{0 \theta}^{\mathrm{X}}=\overline{I_{0}} A_{0}+0.5 I_{1} A_{1} \cos \psi, & I_{0 \theta}^{\mathrm{Y}}=0 \\ I_{1 \theta}^{\mathrm{X}}=\bar{I}_{0} A_{1}+I_{1} \cos \psi\left(A_{0}+0.5 A_{2}\right) & \\ I_{1 \theta}^{\mathrm{Y}}=I_{1} \sin \psi\left(A_{0}-0.5 A_{2}\right) & \\ I_{k \theta}^{\mathrm{X}}=I_{0} A_{k}+0.5 I_{1} \cos \psi\left(A_{k-1}+A_{k+1}\right) & \\ I_{k \theta}^{\mathrm{Y}}=0.5 I_{1} \sin \psi\left(A_{k-1}-A_{k+1}\right), & \end{cases}
$$

where $A_{k}$ are the $k$-th order Fourier components of the transmittance, $A_{k}=P S(T) L c H_{k}$ in which the $H_{k}$ is the $k$-th order Fourier components of the spectral line shape. In LIA, the $k$-th Fourier components can be fixed by the same frequency reference signals as:

$\left\{\begin{array}{l}\mathrm{X}:\left[I_{k \theta}^{\mathrm{X}} \cos k \theta-I_{k \theta}^{\mathrm{Y}} \sin k \theta\right] \cos \left(k \omega t+\beta_{k}^{\mathrm{X}}\right) \\ \mathrm{Y}:\left[I_{k \theta}^{\mathrm{X}} \cos k \theta-I_{k \theta}^{\mathrm{Y}} \sin k \theta\right] \sin \left(k \omega t+\beta_{k}^{\mathrm{Y}}\right), \quad k=0,1,2, \cdots .\end{array}\right.$

From Eq. 7, it can be seen that the amplitudes of the harmonic in $\mathrm{X}$ and $\mathrm{Y}$ axes are decided by the relationship between the phase shift in wavelength $(\eta$ or $k \theta)$ and the phases of the reference signal $\left(\beta_{k}^{\mathrm{X}}\right.$ and $\left.\beta_{k}^{\mathrm{Y}}\right)$. Due to the arbitrary of $\eta$, the amplitudes of the harmonic in $\mathrm{X}$ and $\mathrm{Y}$ axes are variable in each measurement. Generally, the magnitude of the Fourier components is used as the complete harmonic signal: $S_{k f}=\sqrt{I_{k \theta}^{\mathrm{X}}+I_{k \theta}^{\mathrm{Y} 2}} \approx \overline{I_{0}} A_{k}$ when the RAM can be neglected. However, this method is invalid in the first harmonic since the RAM is strong. Or when setting the phases of the reference signal: $\beta_{k}^{\mathrm{X}}=\beta_{k}^{\mathrm{Y}}=k \eta$, the $k$-th Fourier components can accumulate on their own axes as:

$$
\left\{\begin{array}{l}
X_{k f}=I_{k \theta}^{\mathrm{X}} \\
Y_{k f}=I_{k \theta}^{\mathrm{Y}}
\end{array}\right.
$$

\section{APPENDIX B}

\section{MODULATION INDEX}

The amplitudes of the first, second and third harmonics vary with the modulation index $(\mathrm{m})$ are calculated as shown in Fig. 10 and Table II, where the gas parameters are the same as Fig. 5. As indicated in the figure and table, when $m=2.0$ or 2.2 , the first and second harmonics would reach to their maximum values while the amplitude of the third harmonic is only $88 \%$ of its maximum. When $m$ increases to 3.0 , the percentage of the third harmonic will raise to

46

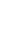

2

4

6

(

9




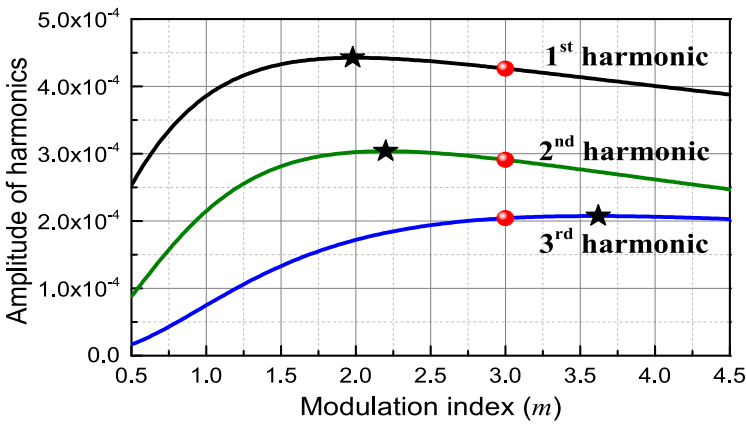

Fig. 10. The amplitudes of the first, second and third harmonics vary with modulation index $(\mathrm{m})$ from 0.5 to 4.5 . The black stars denote the maximum at each harmonics and the red dots are the harmonic amplitudes at $m=3.0$.

TABLE II

OVERVIEW OF THE HARMONICS AT DIFFERENT MODULATION INDICES, the Percentage Data Are the Harmonics Amplitudes COMPARED With THEIR OWN MAXIMUM $\left(m_{\mathrm{MAX}}\right)$

\begin{tabular}{c|ccc}
\hline \hline & $S_{1 f}$ & $S_{2 f}$ & $S_{3 f}$ \\
\hline$m_{\max }$ & 2.0 & 2.2 & 3.6 \\
$m=2.2$, percent of maximum & $99.8 \%$ & $100 \%$ & $88.0 \%$ \\
$m=3.0$, percent of maximum & $96.4 \%$ & $95.8 \%$ & $98.4 \%$ \\
\hline \hline
\end{tabular}

$98.4 \%$, while the amplitudes of the first and second harmonics are still $>95 \%$ of their maximums. When setting $m=3.0$, the high SNR harmonics, especially the third harmonic, will be obtained.

\section{APPENDIX C \\ MODULATION DEPTH}

According to Eq. 4, the instantaneous wavenumber can also be expressed as determined by the tuning current $(i)$ :

$$
v(i)=c_{2}\left(i-i_{0}\right)^{2}+c_{1}\left(i-i_{0}\right)+c_{0},
$$

where $i$ is transferred from the tuning voltage (ramp signal) and the voltage-to-current is determined by the laser controller ( $5 \mathrm{~mA} / \mathrm{V}$ in the system); $i_{0}$ is the fixed current which can be set as the current at the line centers. The coefficients $\left(c_{2}, c_{1}\right.$, and $c_{0}$ ) can be inferred from the measured line centers. Then, the modulation depth can be calculated by:

$$
a=\frac{d v(i)}{d i} I_{1} \varsigma(\omega)=\left[2 c_{2}\left(i-i_{0}\right)+c_{1}\right] I_{1} \varsigma(\omega),
$$

where $\varsigma(\omega)$ is the frequency dependency of the current-towavenumber tuning coefficient, the value can be seen in [29] and [48].

\section{ACKNOWLEDGMENT}

The authors gratefully acknowledge VERTILAS GmbH for kindly providing the VCSEL laser in the TDLAS measurement system and thank Dr. Christian Neumeyr and Jürgen Rosskopf for the valuable technical consultation and input. They want to thank Sheng Ye and Dr. Mark Wenig from Ludwig Maximilian University of Munich (LMU) for providing the Li-Cor 840A $\mathrm{CO}_{2} / \mathrm{H}_{2} \mathrm{O}$ gas analyzer and Dr. Stephan Hachinger from Leibniz Supercomputing Center (Leibniz-Rechenzenturm, LRZ) for helping the HYSPLIT trajectory calculation. They also thank Dr. Yingchun Wu from Zhejiang University, Yonglu Zhu from Beijing Research Institute of Telemetry, and Yanfang Li from Shandong Academy of Science, China for supporting assistance on designing the experimental setup.

\section{REFERENCES}

[1] R. K. Pachauri et al., "Climate change 2014: Synthesis report. Contribution of working groups I, II and III to the fifth assessment report of the intergovernmental panel on climate change," Intergovernmental Panel Climate Change, Geneva, Switzerland, Tech. Rep., 2014.

[2] F. M. Bréon et al., "An attempt at estimating Paris area $\mathrm{CO}_{2}$ emissions from atmospheric concentration measurements," Atmos. Chem. Phys., vol. 15, no. 4, pp. 1707-1724, 2015.

[3] K. McKain, S. C. Wofsy, T. Nehrkorn, J. Eluszkiewicz, J. R. Ehleringer, and B. B. Stephens, "Assessment of ground-based atmospheric observations for verification of greenhouse gas emissions from an urban region," Proc. Nat. Acad. Sci. USA, vol. 109, no. 22, pp. 8423-8428, 2012.

[4] J. Chen et al., "Differential column measurements using compact solar-tracking spectrometers," Atmos. Chem. Phys., vol. 16, no. 13 , pp. 8479-8498, 2016.

[5] F. J. Duarte, Tunable Lasers Handbook. Amsterdam, The Netherlands: Elsevier, 1996.

[6] J. Jiang et al., "TDLAS-based detection of dissolved methane in power transformer oil and field application," IEEE Sensors J., vol. 18, no. 6 , pp. 2318-2325, Mar. 2018.

[7] C. Liu, Z. Cao, Y. Lin, L. Xu, and H. McCann, "Online cross-sectional monitoring of a swirling flame using TDLAS tomography," IEEE Trans. Instrum. Meas., vol. 67, no. 6, pp. 1338-1348, Jun. 2018.

[8] J. Reid and D. Labrie, "Second-harmonic detection with tunable diode lasers-Comparison of experiment and theory," Appl. Phys. B, vol. 26, no. 3, pp. 203-210, 1981

[9] J. Chen, A. Hangauer, R. Strzoda, and M.-C. Amann, "Laser spectroscopic oxygen sensor using diffuse reflector based optical cell and advanced signal processing," Appl. Phys. B, vol. 100, no. 2, pp. 417-425, 2010.

[10] C. Liu and L. Xu, "Laser absorption spectroscopy for combustion diagnosis in reactive flows: A review," Appl. Spectrosc. Rev., 2018. doi: $10.1080 / 05704928.2018 .1448854$.

[11] A. Hangauer, J. Chen, R. Strzoda, and M.-C. Amann, "Multi-harmonic detection in wavelength modulation spectroscopy systems," Appl. Phys. $B$, vol. 110, no. 2, pp. 177-185, 2013.

[12] L. J. Lan, Y. J. Ding, Z. M. Peng, Y. J. Du, Y. F. Liu, and Z. Li, "Multiharmonic measurements of line shape under low absorption conditions," Appl. Phys. B, vol. 117, no. 2, pp. 543-547, 2014.

[13] H. Li, G. B. Rieker, X. Liu, J. B. Jeffries, and R. K. Hanson, "Extension of wavelength-modulation spectroscopy to large modulation depth for diode laser absorption measurements in high-pressure gases," Appl. Opt., vol. 45, no. 5, pp. 1052-1061, Feb. 2006.

[14] P. Zhimin, D. Yanjun, C. Lu, L. Xiaohang, and Z. Kangjie, "Calibrationfree wavelength modulated TDLAS under high absorbance conditions," Opt. Express, vol. 19, no. 23, pp. 23104-23110, 2011

[15] L. J. Lan, Y. J. Ding, Z. M. Peng, Y. J. Du, and Y. F. Liu, "Calibrationfree wavelength modulation for gas sensing in tunable diode laser absorption spectroscopy," Appl. Phys. B, vol. 117, no. 4, pp. 1211-1219, 2014.

[16] A. J. McGettrick, K. Duffin, W. Johnstone, G. Stewart, and D. G. Moodie, "Tunable diode laser spectroscopy with wavelength modulation: A phasor decomposition method for calibration-free measurements of gas concentration and pressure," J. Lightw. Technol., vol. 26, no. 4, pp. 432-440, Feb. 15, 2008.

[17] W. Johnstone, A. J. McGettrick, K. Duffin, A. Cheung, and G. Stewart, "Tunable diode laser spectroscopy for industrial process applications: System characterization in conventional and new approaches," IEEE Sensors J., vol. 8, no. 7, pp. 1079-1088, Jul. 2008.

[18] G. Stewart, W. Johnstone, J. R. P. Bain, K. Ruxton, and K. Duffin, "Recovery of absolute gas absorption line shapes using tunable diode laser spectroscopy with wavelength modulation-Part I: Theoretical analysis," J. Lightw. Technol., vol. 29, no. 6, pp. 811-821, Mar. 15, 2011.

[19] T. Benoy, M. Lengden, G. Stewart, and W. Johnstone, "Recovery of absorption line shapes with correction for the wavelength modulation characteristics of DFB lasers," IEEE Photon. J., vol. 8, no. 3, Jun. 2016, Art. no. 1501717.

[20] D. M. Sonnenfroh et al., "Application of balanced detection to absorption measurements of trace gases with room-temperature, quasi-cw quantum-cascade lasers," Appl. Opt., vol. 40, no. 6, pp. 812-820, 2001.

[21] A. L. Chakraborty, K. Ruxton, W. Johnstone, M. Lengden, and K. Duffin, "Elimination of residual amplitude modulation in tunable diode laser wavelength modulation spectroscopy using an optical fiber delay line," Opt. Express, vol. 17, no. 12, pp. 9602-9607, 2009. 
[22] J. R. P. Bain, M. Lengden, G. Stewart, and W. Johnstone, "Recovery of absolute absorption line shapes in tunable diode laser spectroscopy using external amplitude modulation with balanced detection," IEEE Sensors J., vol. 16, no. 3, pp. 675-680, Feb. 2016.

[23] P. Zhimin, D. Yanjun, C. Lu, and Y. Qiansuo, "Odd harmonics with wavelength modulation spectroscopy for recovering gas absorbance shape," Opt. Express, vol. 20, no. 11, pp. 11976-11985, 2012.

[24] A. Hangauer, J. Chen, R. Strzoda, M. Ortsiefer, and M.-C. Amann, "Wavelength modulation spectroscopy with a widely tunable InP-based $2.3 \mu \mathrm{m}$ vertical-cavity surface-emitting laser," Opt. Lett., vol. 33, no. 14 , pp. 1566-1568, 2008.

[25] J. Chen et al., " $\mathrm{CO}$ and $\mathrm{CH}_{4}$ sensing with single mode $2.3 \mu \mathrm{m} \mathrm{GaSb}-$ based VCSEL," in Proc. Conf. Lasers Electro-Opt. Conf. Quantum Electron. Laser Sci. Conf., Jun. 2009, pp. 1-2.

[26] A. Hangauer, J. Chen, and M.-C. Amann, "Vertical-cavity surfaceemitting laser light-current characteristic at constant internal temperature," IEEE Photon. Technol. Lett., vol. 23, no. 18, pp. 1295-1297, Sep. 15, 2011

[27] Y. Wang et al., "Tunable diode laser absorption spectroscopy-based detection of propane for explosion early warning by using a vertical cavity surface enhanced laser source and principle component analysis approach," IEEE Sensors J., vol. 17, no. 15, pp. 4975-4982, Aug. 2017.

[28] A. Hangauer, J. Chen, K. Seemann, P. Karge, R. Strzoda, and M.-C. Amann, "Compact VCSEL-based $\mathrm{CO}_{2}$ and $\mathrm{H}_{2} \mathrm{O}$ sensor with inherent wavelength calibration for safety and air-quality applications," in Proc. Conf. Lasers Electro-Opt., Opt. Soc. Amer, 2010, p. JThB3.

[29] J. Chen, A. Hangauer, R. Strzoda, and M. Amann, "VCSEL-based calibration-free carbon monoxide sensor at $2.3 \mu \mathrm{m}$ with in-line reference cell," Appl. Phys. B, vol. 102, no. 2, pp. 381-389, 2011.

[30] L. Lan, J. Chen, Y. Wu, Y. Bai, X. Bi, and Y. Li, "Self-Calibrated Multiharmonic $\mathrm{CO}_{2}$ sensor using VCSEL for urban in situ measurement," IEEE Trans. Instrum. Meas., vol. 68, no. 4, pp. 1140-1147, 2019.

[31] P. Zhimin, D. Yanjun, J. Junwei, L. Lijuan, D. Yanjun, and L. Zheng, "First harmonic with wavelength modulation spectroscopy to measure integrated absorbance under low absorption," Opt. Express, vol. 21, no. 20, pp. 23724-23735, 2013.

[32] J. Chen, "Compact laser-spectroscopic gas sensors using vertical-cavity surface-emitting lasers," Ph.D. dissertation, Dept. Elect. Comput. Eng., Technical Univ. Munich, Munich, Germany, 2011.

[33] Vertilas. Products. Accessed: 29, May 2018. [Online]. Available: https://vertilas.com/content/nir-vcsels-gas-analysis-tdlas

[34] A. Savitzky and M. J. E. Golay, "Smoothing and differentiation of data by simplified least squares procedures," Anal. Chem., vol. 36, no. 8, pp. 1627-1639, 1964.

[35] J. Chen, A. Hangauer, R. Strzoda, and M.-C. Amann, "Tunable diode laser spectroscopy with optimum wavelength scanning," Appl. Phys. B, vol. 100 , no. 2 , pp. $331-339,2010$

[36] L. S. Rothman et al., "The HITRAN 2008 molecular spectroscopic database," J. Quant. Spectrosc. Radiat. Transf., vol. 110, nos. 9-10, pp. 533-572, 2009.

[37] D. W. Allan, "Statistics of atomic frequency standards," Proc. IEEE, vol. 54, no. 2, pp. 221-230, Feb. 1966

[38] P. Werle, R. Mücke, and F. Slemr, "The limits of signal averaging in atmospheric trace-gas monitoring by tunable diode-laser absorption spectroscopy (TDLAS)," Appl. Phys. B, vol. 57, no. 2, pp. 131-139, 1993.

[39] Y. Tohjima et al., "Theoretical and experimental evaluation of the isotope effect of NDIR analyzer on atmospheric $\mathrm{CO}_{2}$ measurement," J. Geophys. Res., Atmos., vol. 114, Jun. 2009, Art. no. D13302.

[40] R. B. Stull, An Introduction to Boundary Layer Meteorology, vol. 13. London, U.K.: Springer, 2012.

[41] A. G. Williams et al., "Radon as a tracer of atmospheric influences on traffic-related air pollution in a small inland city," Tellus B, Chem. Phys. Meteorol., vol. 68, no. 1, 2016, Art. no. 30967.

[42] A. Stohl, "Trajectory statistics-A new method to establish sourcereceptor relationships of air pollutants and its application to the transport of particulate sulfate in Europe," Atmos. Environ., vol. 30, no. 4, pp. $579-587,1996$.

[43] L. L. Ashbaugh, W. C. Malm, and W. Z. Sadeh, "A residence time probability analysis of sulfur concentrations at Grand Canyon National Park," Atmos. Environ., vol. 19, no. 8, pp. 1263-1270, 1985.

[44] H. Ghasemifard et al., "Atmospheric $\mathrm{CO}_{2}$ and $\delta^{13} \mathrm{C}$ measurements from 2012 to 2014 at the Environmental Research Station Schneefernerhaus, Germany: Technical corrections, temporal variations and trajectory clustering," Aerosol Air Qual. Res., vol. 19, pp. 657-670, 2019.
[45] D. C. Carslaw and K. Ropkins, "Openair-An R package for air quality data analysis," Environ. Model. Softw., vols. 27-28, pp. 52-61, 2012.

[46] N. J. Pekney, C. I. Davidson, L. Zhou, and P. K. Hopke, "Application of PSCF and CPF to PMF-modeled sources of $\mathrm{PM}_{2.5}$ in Pittsburgh," Aerosol Sci. Technol., vol. 40, no. 10, pp. 952-961, 2006.

[47] Y. Liu, J. Lin, G. Huang, Y. Guo, and C. Duan, "Simple empirical analytical approximation to the Voigt profile," J. Opt. Soc. Amer. B, vol. 18 , no. 5 , pp. $666-672,2001$.

[48] J. Chen, A. Hangauer, R. Strzoda, and M.-C. Amann, "Experimental characterization of the frequency modulation behavior of vertical cavity surface emitting lasers," Appl. Phys. Lett., vol. 91, no. 14, 2007, Art. no. 141105 .

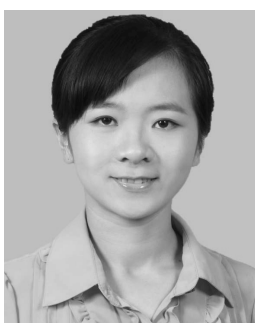

Lijuan Lan received the B.Sc. degree from the Department of Precision Instrument, Tsinghua University, Beijing, China, in 2012, and the M.Eng. degree from the Department of Thermal Engineering from Tsinghua University in 2015 . She is currently pursuing the Ph.D. degree in environmental sensing and modeling with the Technical University of Munich, Germany. Her current research interests include the in situ GHG detection based on the optical techniques and the air parcel backward trajectory modeling in urban areas.

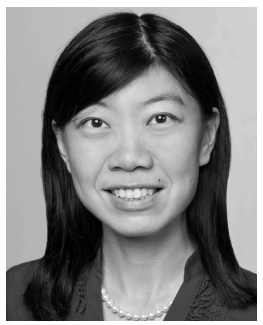

Jia Chen (M'08) received the Diploma degree from the University of Karlsruhe, Germany, in 2006, and the Ph.D. degree from the Technical University of Munich (TUM) in 2011. From 2011 to 2015, she was a Post-Doctoral Fellow with the Environmental Science and Engineering Department, Harvard University. In 2015, she was appointed as a Professor at TUM, where she is the Head of the Environmental Sensing and Modeling Group, Department of Electrical and Computer Engineering. She is also an Associate with the Department of Earth and Planetary Sciences, Harvard University. She has authored and co-authored 34 peer-reviewed journals and 85 conference publications, and has filed 12 patents.

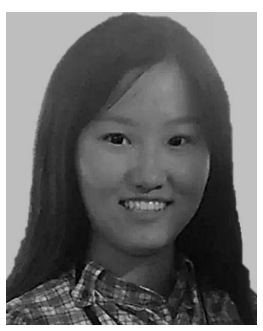

Xinxu Zhao received the B.Eng. degree in thermal energy and power engineering in 2015 and the M.Sc. degree in international joint master programme sustainable development from Utrecht University, The Netherlands, and Leipzig University, Germany, in 2017. She is currently pursuing the $\mathrm{Ph} . \mathrm{D}$. degree in environmental sensing and modeling with the Technical University of Munich, Germany. Her research interests include multi-scale atmospheric modelings, data analysis of GHG emissions and air pollutants between measurements and modelings, and column and in situ measurements of GHG.

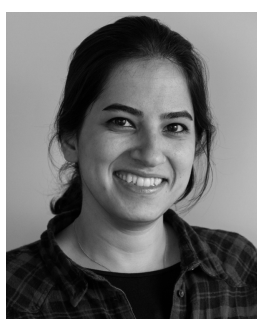

Homa Ghasemifard received the B.Sc. degree in physics from the Plasma Physics Research Center, Azad University, Tehran, Iran, in 2009, and the M.Sc. degree in environmental physics from Bremen University, Germany, in 2013. She is currently pursuing the Ph.D. degree with the Professorship of Ecoclimatology Department, Technical University of Munich, Germany, working on GHG measurements and pollution events at the remote high elevated research station Schneefernerhaus in Germany. She is a Researcher with the Technical University of Munich working on backward trajectories' simulation. 